Revue d'histoire de l'Amérique française

Q4. REVUE D.HISTOIRE DE L'AMÉRIQUE FRANÇAISE

\title{
Réflexions sur l'histoire des femmes dans l'histoire du Québec
}

\section{Andrée Lévesque}

Volume 51, numéro 2, automne 1997

Les pratiques de l'histoire de l'Amérique française depuis 50 ans

URI : https://id.erudit.org/iderudit/305649ar

DOI : https://doi.org/10.7202/305649ar

Aller au sommaire du numéro

\section{Éditeur(s)}

Institut d'histoire de l'Amérique française

\section{ISSN}

0035-2357 (imprimé)

1492-1383 (numérique)

Découvrir la revue

\section{Citer cet article}

Lévesque, A. (1997). Réflexions sur l'histoire des femmes dans l'histoire du Québec. Revue d'histoire de l'Amérique française, 51(2), 271-284.

https://doi.org/10.7202/305649ar

\section{Résumé de l'article}

Cette réflexion sur l'impact de l'histoire des femmes sur l'histoire du Québec témoigne de la redéfinition de l'histoire traditionnelle par les interrogations et les débats soulevés par l'histoire des femmes. Celle-ci, inspirée par le mouvement féministe et encouragée par la nouvelle histoire sociale, s'est, dès ses débuts, ouverte à l'inter et à la multidisciplinarité. Des problématiques inédites et de nouveaux sujets sont apparus, par exemple, les rapports sociaux de sexe, la socialisation, la reproduction, la sexualité, le travail non rémunéré. Jusqu'ici, ces thèmes ont surtout été abordés en histoire de la classe ouvrière et en histoire de la famille. Si les historiennes spécialisées en histoire des femmes ont inclu ces sujets dans leur enseignement et dans leur recherche sur des thèmes plus généraux, la lenteur de certains de leurs collègues à intégrer les femmes n'est, nous l'espérons, qu'une question de temps et surtout de génération. 


\title{
RÉFLEXIONS SUR L'HISTOIRE DES FEMMES DANS L'HISTOIRE DU QUÉBEC
}

\author{
ANDRÉE LÉVESQUE \\ Département d'histoire \\ Université McGill
}

\section{RÉSUMÉ}

Cette réflexion sur l'impact de l'histoire des femmes sur l'histoire du Québec témoigne de la redéfinition de l'histoire traditionnelle par les interrogations et les débats soulevés par l'histoire des femmes. Celle-ci, inspirée par le mouvement féministe et encouragée par la nouvelle histoire sociale, s'est, dès ses débuts, ouverte à l'inter et à la multidisciplinarité. Des problématiques inédites et de nouveaux sujets sont apparus, par exemple, les rapports sociaux de sexe, la socialisation, la reproduction, la sexualité, le travail non rémunéré. Jusqu'ici, ces thèmes ont surtout été abordés en histoire de la classe ouvrière et en histoire de la famille. Si les historiennes spécialisées en histoire des femmes ont inclu ces sujets dans leur enseignement et dans leur recherche sur des thèmes plus généraux, la lenteur de certains de leurs collègues à intégrer les femmes n'est, nous l'espérons, qu'une question de temps et surtout de génération.

\section{ABSTRACT}

One notes the impact of women's history on Québec history in the new questionings and debates that are leading to a redefinition of traditional history. This new way of looking at Québec history has its origin in the feminist movement of the last 25 years and has been encouraged by the new social history. One of its contribution is its openness to interdisciplinarity. Novel approaches and subjects are now part of this field of enquiry: gender relations, socialisation, reproduction, sexuality, unpaid work have all recently been the subjects of books or articles. Labour history and family are the two fields that have proved particularly open to these themes. If historians specialized in women's history have included these topics in their teaching and in their research on more general subjects, one has to look at the teaching, research and publications of non-specialists give us the measure of the penetration of women's history in the broader Quebec history. The slowness of certain colleagues to integrate women in their research and teaching is, we hope, only a question of generation. 
Depuis une vingtaine d'années, les oubliés de l'histoire ont secoué la discipline historique par de nouveaux questionnements et par de nouvelles exigences. Ce mouvement de renouvellement lié à la Nouvelle gauche des années 1960 et à l'insistance des sujets de l'histoire pour s'exprimer, a vu l'éclosion non seulement de champs d'investigation jusqu'alors inexplorés, mais aussi d'interrogations et de problématiques inédites. Afin de situer l'évolution de l'histoire des femmes dans la foulée des pratiques renouvelées de l'histoire ou de ce qu'on a appelé la Nouvelle histoire', il convient de retracer les débuts de cette spécialisation dans notre discipline. Le présent article n'est pas une bibliographie, il en existe d'excellentes ${ }^{2}$. Il n'est pas non plus un bilan historiographique de tout ce qui s'est écrit sur la question depuis plus de vingt ans. Il se propose plutôt de réfléchir sur les relations entre l'histoire des femmes et l'histoire du Québec, afin d'identifier certains lieux de rencontre dans la recherche et dans l'enseignement et de souligner les mécanismes d'intégration d'un nouveau champ de la connaissance. Pour ce faire, il faut se reporter trente ans en arrière.

Issue du mouvement et de l'idéologie féministes amorcés en Occident à la fin des années 1960, et liée à l'expansion et à la redéfinition de la nouvelle histoire sociale, l'histoire des femmes s'est constamment développée et transformée depuis presque trente ans ${ }^{3}$. Les féministes de la fin de la deuxième vague ${ }^{4}$ se sont interrogées, au Québec comme ailleurs, sur l'origine de leur oppression, sur ses différentes manifestations dans le temps, sur leur propre histoire. Parmi ces militantes se trouvaient des historiennes prêtes à orienter leur recherche vers la découverte de leur passé collectif. D'autres historiennes firent

1. Les historiographes francophones de la Nouvelle histoire ont mis du temps à reconnaître cette nouvelle venue. En 1974, Jacques Le Goff et Pierre Nora dirigeaient une collection en trois volumes sur les «nouveaux problèmes, nouvelles approches et nouveaux objets», sans trouver place dans leur «échantillonnage significatif» pour l'histoire des femmes. J. Le Goff et P. Nora, dir., Faire de l'histoire (Paris, Gallimard, 1974), 3 vol. Quatre ans plus tard, dans La nouvelle histoire, Cécile Dauphin signait quatre pages sur l'histoire des femmes. Jacques Le Goff, La nouvelle histoire (Paris, Retz, 1978).

2. Diana Pedersen, Changing Women, Changing History: a Bibliography of Women in Canada (Ottawa, Carleton University Press, 1996), comprend quelques centaines de titres sur le Québec. On y trouve aussi une liste des bibliographies et des bilans historiographiques en histoire des femmes qu'il ne convient pas d'énumérer ici.

3. On a fait plus d'une fois la genèse de l'histoire des femmes. Voir entre autres, Denise Angers et Chistine Piette, «Critique féministe et histoire», dans Roberta Mura. dir., Critiques féministes des disciplines (Sainte-Foy, Cahiers de recherche du GREMF, 1988), I: 7-21. Denyse Baillargeon, «Des voies/x parallèles. L'histoire des femmes au Québec et au Canada anglais», Sextant, 4, (1995): 133-168.

4. Les féministes de la première vague se situent à la fin du XIX $\mathrm{X}^{\mathrm{e}}$ et au début du $\mathrm{XX}^{\mathrm{e}}$ siècle, celles de la deuxième vague reprirent le flambeau à la fin des années 1960 . 
le cheminement inverse et devinrent féministes en s'interrogeant sur leur histoire. C'était le début d'un nouveau champ de recherche.

Centrée sur les politiciens et les grands événements, l'histoire traditionnelle ne pouvait intégrer ces sujets innovateurs. Cette ancienne histoire officielle était déjà pourfendue dans la $R H A F$, en juin 1955, par Claude Galarneau qui, sur les traces de Marc Bloch, réclamait une histoire des «hommes vivant en groupes ${ }^{5} »$. Nulle part dans l'article n'est-il fait état que l'humanité se compose de deux sexes pour qui les «événements», les «faits», c'est-à-dire le passé dont l'histoire fait le «récit», sont spécifiques à chacun. Ainsi définie, l'histoire s'avérait une discipline inadéquate pour inclure et expliquer l'expérience de plus de la moitié des citoyens. Il fallut attendre les praticiens et praticiennes de l'histoire sociale, quelques années plus tard, pour accueillir l'histoire des femmes; mais cette dernière, irréductible à son mentor, eut tôt fait d'en déborder les limites. Ainsi, les études sur les droits civils et sur le suffrage la rattachent à l'histoire politique; celles sur le travail et sur la contribution des femmes au développement économique l'associent à l'histoire économique; en faisant l'histoire des femmes, on peut toucher aussi bien à l'histoire militaire qu'à l'histoire religieuse, comme à toutes les branches de l'histoire sociale.

L'historienne américaine Gerda Lerner, dans un des premiers articles historiographiques consacré à l'histoire des femmes, écrit, en simplifiant beaucoup, que celle-ci s'est d'abord intéressée aux biographies de femmes célèbres, puis à l'étude de leurs institutions. Dans un premier temps, selon Lerner, les historiennes féministes ont cherché à compenser l'absence de femmes dans l'histoire en débusquant les oubliées; puis, dans un deuxième temps, elles se sont penchées sur les apports des femmes à l'histoire telle que définie par les hommes sur le rôle des femmes dans l'élargissement de la démocratie, par exemple - , pour enfin inclure dans leur pratique historienne des thèmes rarement abordés auparavant ${ }^{6}$. C'est ainsi qu'il fut possible d'étudier légitimement la maternité et la contraception, et tout ce qui se rattache à ce qu'il est convenu d'appeler la sphère privée.

L'historiographie québécoise a suivi une trajectoire quelque peu différente. Peut-être à cause de l'influence de l'Eglise catholique depuis les débuts de la colonie et du rôle, subordonné mais essentiel, des femmes dans l'Église, celles-ci n'ont jamais été complètement absentes de l'histoire de la Nouvelle-France: de Jeanne Mance à Madeleine

5. Claude Galarneau, «Jeunesse de Clio ou la recherche en histoire», $R H A F, 11,1$ (juin 1955): 3-13.

6. Gerda Lerner, The Majority Finds Its Past: Placing Women in History (New York, Oxford University Press, 1979). 
de Verchères en passant par Marguerite Bourgeoys, Kateri Tekakouitha et Marie de l'Incarnation, elles ont inspiré de nombreux articles, plus ou moins hagiographiques, au cours des années ${ }^{7}$. Il a cependant fallu attendre les années 1970 pour qu'apparaisse une histoire ayant pour objet non pas des personnalités exemplaires, mais la condition féminine dans son ensemble. Un des premiers écrits en histoire des femmes, conçu dans une approche féministe, c'est-à-dire centré sur l'expérience féminine et les rapports sociaux de sexes, est probablement celui de Micheline Dumont, publié en 1970, pour la commission Bird: «L'histoire de la condition de la femme dans la province de Québec ${ }^{8}$ ». Dans un pays de code civil, il n'est pas étonnant que les premières recherches se soient penchées sur les fondements juridiques de l'infériorisation des femmes.

Dans les années 1970, les historiennes - il faut employer le féminin puisque la plupart des chercheurs en histoire des femmes ont été des femmes ${ }^{9}$ - se sont tournées vers les premières féministes et les institutions féminines et féministes. En 1972, Michèle Jean organise un cours sur l'histoire des Québécoises de 1900 à 1960 au Service d'éducation permanente de l'Université de Montréal. Elle puise dans des textes de Québécoises et aussi dans ceux de personnages aussi divers que $\mathrm{M}^{\mathrm{gr}}$ Louis-Adolphe Paquet ou Jacques Grand'Maison qui se sont prononcés sur le rôle des femmes. En 1974, à partir de ce cours, elle publie son recueil Québécoises $d u 20^{e}$ siècle $^{10}$. On y apprend que le Québec a aussi eu ses réformatrices sociales et ses suffragistes dans la Fédération nationale Saint-Jean-Baptiste, l'Alliance pour le vote des femmes et l'Association pour le suffrage féminin ${ }^{11}$. L'année suivante, dans la RHAF, Micheline Dumont se demande: «Peut-on faire l'histoire des femmes?» On trouve dans cet article une mise en garde contre l'utilisation des «critères du présent» pour «évaluer les réalités du passé», suivie d'un bilan de quelques ouvrages récents ${ }^{12}$.

7. Dès sa deuxième année, la Revue d'histoire de l'Amérique française publiait trois articles sur l'«Itinéraire spirituel de Marguerite Bourgeoys» par Yves Charron, pss, RHAF, 2 (1948): 230-237, 351-374, 522-539.

8. Micheline Dumont, «L'histoire de la condition de la femme dans la province de Québec», Tradition culturelle et histoire politique de la femme au Canada (Ottawa, Information Canada, Commission royale d'enquête sur la situation de la femme au Canada (commission Bird), Étude no 8, 1970).

9. Il faut noter une exception: Sylvio Dumas, Les Filles $d u$ Roi en Nouvelle-France (Québec, Société historique de Québec, cahier 24, 1972).

10. Michèle Jean, Québécoises du $20^{e}$ siècle (Montréal, Éditions du Jour, 1974).

11. Marie Lavigne, Yolande Pinard et Jennifer Stoddart, «La Fédération nationale SaintJean-Baptiste et les revendications féministes au début du $20^{\circ}$ siècle», RHAF, 29,3 (décembre 1975): 353-373.

12. Micheline Dumont-Johnson, «Peut-on faire l'histoire des femmes?», RHAF, 29,3 (1975): 421-428. 
Préoccupées du processus de construction de $L a$ femme - «on ne naît pas femme, on le devient», écrivait Simone de Beauvoir, - des historiennes se sont interrogées sur l'éducation des filles ${ }^{13}$. L'éducation devait révéler l'œuvre de socialisation spécifique à un sexe et le rôle de ses agentes les plus actives, les religieuses. Reprenant les mêmes outils qu'elles avaient appris à manier dans leur recherche conventionnelle, des historiennes repérèrent la présence des femmes dans les sources traditionnelles. Les premières recherches reprirent les sources éprouvées, les recensements, les commissions royales d'enquête qui, relues dans une optique féministe, purent fournir des données sexuées longtemps ignorées. Ainsi, une relecture des statistiques officielles a permis de retracer l'évolution du travail des femmes ${ }^{14}$. L'expérience féminine ne pouvait plus désormais être assimilée à l'expérience masculine, qu'il s'agisse de la colonisation en NouvelleFrance ou du chômage pendant les années 1930.

L'expérience des femmes n'a toujours formé qu'un volet de ces premiers travaux. Depuis le début, les discours sur les femmes ont aussi fait partie de la recherche féministe, parfois même aux dépens de l'étude des pratiques réelles. Une abondante littérature ne demandait qu'à être exploitée pour révéler les discours et les prescriptions dont les femmes ont fait l'objet. Un journaliste comme Henri Bourassa, des juristes comme ceux qui siégeaient à la commission Dorion sur la réforme du code civil, des membres du clergé, des médecins et des politiciens ont laissé un corpus abondant et largement univoque sur le rôle des Québécoises ${ }^{15}$. Études empiriques sur les pratiques et les comportements, analyses de discours prescriptifs, la recherche s'intensifie, si bien qu'au programme du congrès de l'IHAF de 1979, on compte sept communications sur les femmes dans l'histoire. De plus en plus d'articles se retrouvent dans les pages des revues savantes, telles la RHAF et Histoire sociale. Au début des années 1980, on peut

13. Nadia Fahmy-Eid et Micheline Dumont, dir., Maîtresses de maison, maîtresses d'école: femmes, familles et éducation dans l'histoire du Québec (Montréal, Boréal Express, 1983). Nicole Thivierge, Écoles ménagères et instituts familiaux: un modèle féminin traditionnel (Québec, Institut québécois de recherche sur la culture, 1982).

14. Marie Lavigne et Jennifer Stoddart, «Les travailleuses montréalaises, 1920-1940", Labour/Le travailleur, 2 (1977): 170-183, repris et élaboré dans Marie Lavigne et Yolande Pinard, dir., Les femmes dans la société québécoise (Montréal, Boréal Express, 1977), 125-145.

15. Susan Mann-Trofimenkoff, «Henri Bourassa and the "Woman Question"», Journal of Canadian Studies/Revue d'études canadiennes, 10,4 (novembre 1975), traduit et publié dans Lavigne et Stoddart, loc. cit., 109-124. Jennifer Stoddart, «Quand des hommes de robe se penchent sur les droits des femmes: le cas de la commission Dorion, 1929-1931», dans David H. Hamilton, dir., Essays in the History of Canadian Law (Toronto, The Osgoode Society, University of Toronto Press, 1981), vol. 2, traduit et reproduit dans Lavigne et Pinard, loc. cit., 307336. 
lire les premiers bilans historiographiques sur une décennie de recherches en histoire des femmes ${ }^{16}$.

Indissociables, l'enseignement et la recherche s'inspirent mutuellement pour enrichir notre connaissance du passé. L'enseignement en histoire des femmes a été à la fois la conséquence de l'offre et de la demande. Dans certaines institutions, des historiennes féministes qui, à cette époque, n'avaient pas été formées en histoire des femmes mais avaient dû se recycler dans ce domaine, ont proposé de tels cours ${ }^{17}$. Par ailleurs, des étudiantes ont insisté, dans d'autres institutions, pour apprendre l'histoire de leur genre. On a donc vu des directeurs de département faire appel à une de leurs (quelques) collègues féminines et lui demander de bien vouloir offrir un cours en histoire des femmes au Québec ou au Canada. Par la force des choses, des professeures ont ainsi découvert toute une histoire qu'elles avaient jusque-là à peu près ignorée. Enfin, dans des institutions plus lentes à innover, des historiennes se sont emparées de cours déjà au programme, en histoire sociale par exemple, et ont accordé aux femmes la parité. Dans tous ces cas, sans guide ni repère, en cherchant directement dans les sources, des historiennes ont offert un enseignement qui a immédiatement attiré des étudiantes et aussi des étudiants.

Des historiens se sont montrés curieux à l'endroit de ce nouveau champ, certains y ont même vu une occasion de renouveler leurs cours. Je me souviens d'un collègue qui, apprenant que j'allais offrir un cours en histoire des femmes, m'a répondu: «Très intéressant. Je devrais aussi m'y mettre: cette année dans mon cours d'histoire sociale, je fais la mort, l'année prochaine je pourrais enseigner les femmes.» Ici, il s'agissait d'un sujet comme un autre. Il n'en était pas de même pour la plupart des historiennes et des étudiantes de la première heure qui, avides de se documenter sur leur passé, se voyaient à la fois objets et sujets de l'histoire.

En 1982 paraît l'ouvrage qui changera la donne de l'enseignement et de la recherche en histoire du Québec: L'histoire des femmes au

16. «Travail et mouvement des femmes: une histoire visible. Bilan historiographique», dans Lavigne et Pinard, loc. cit., 7-60. Nadia Fahmy-Eid et Micheline Dumont, «Les rapports femmes/famille/éducation au Québec: bilan de recherche», dans Fahmy-Eid et Dumont, loc. cit., 5-46; Micheline Dumont, «Découvrir la mémoire des femmes», dans Fahmy-Eid et Dumont, loc. cit., 262-276. Il est significatif qu'en 1983, dans sa bibliographie annuelle, la Canadian Historical Review inaugure une section sur l'histoire des femmes, entre l'histoire économique et l'histoire sociale. Canadian Historical Review, 63,3 (septembre 1983): 427. La RHAF continue de procéder par catégories chronologiques.

17. Micheline Dumont avait soutenu une thèse de doctorat sur les missionnaires en Acadie, Nadia Fahmy-Eid sur les ultramontains, Andrée Lévesque sur la gauche au Québec pendant les années 1930 et Susan Mann-Trofimenkoff sur l'Action française. 
Québec, du collectif $\mathrm{Clio}^{18}$. Quatre pionnières mettent en commun leurs recherches pour élaborer une vaste synthèse de la condition féminine, des structures qui la régissent et de l'expérience des Québécoises depuis la Nouvelle-France jusqu'à la Révolution tranquille. Leurs travaux reflètent l'état de la recherche à cette époque, laissant, certes, béants certains pans d'une histoire encore méconnue, mais traçant aussi des pistes qui devaient inspirer par la suite toute une génération d'historiennes.

Ses origines et ses préoccupations épistémologiques font cependant de l'histoire des femmes une histoire pas comme les autres. Le sujet s'identifiant à l'objet étudié, on accuse cette histoire de subjectivisme, voire de victimologie. On avait déjà fait ce reproche à l'histoire des Afro-Américains en essor à la même époque. Quand des historiennes ont insisté sur le rôle actif des femmes dans la construction de leur destinée, on leur a reproché d'idéaliser leurs sujets. Les critiques et les autocritiques n'ont pas manqué et ont suscité des remises en question et des efforts de conceptualisation qui se poursuivent encore. En 1991, face à un certain scepticisme, Nadia Fahmy-Eid a plaidé la cause de la scientificité possible de l'histoire, précisément en prenant l'histoire des femmes comme exemple ${ }^{19}$.

Ouverte dès le départ à l'interdisciplinarité par le biais du mouvement féministe ou des études sur les femmes, l'histoire des femmes est constamment traversée par des questionnements empruntés à la sociologie, à la science économique ou à la linguistique, ce qui la pousse à adopter une démarche historique liée aux préoccupations de ces disciplines. Le «tournant linguistique», par exemple, a fait l'objet de nombreuses discussions ${ }^{20}$. Longtemps construites elles-mêmes sur un mode essentialiste (femme/femmes), les femmes sont bien placées pour saisir les mécanismes constructionnistes et les rapports de pouvoir qui les sous-tendent.

«Essentialisées» depuis la nuit des temps, autant par l'Église que par Jean-Jacques Rousseau ou, deux siècles plus tard, par Henri Bourassa, les femmes intéressées à leur histoire sont devenues plus sensibles à d'autres types d'essentialisme. Multiples, les femmes se conçoivent et s'écrivent désormais au pluriel: «Les femmes» étudiées ont

18. Collectif Clio, L'Histoire des femmes au Québec depuis quatre siècles (Montréal, Hurtubise HMH, 1982). Les quatre auteures sont: Micheline Dumont, Michèle Jean, Marie Lavigne et Jennifer Stodart. L'ouvrage a donné lieu à une deuxième édition en 1992.

19. Nadia Fahmy-Eid, «Histoire, objectivité et scientificité. Jalons pour une reprise du débat épistémologique», Histoire sociale/Social History, 24,47 (mai 1991): 9-34.

20. Le sujet a fait l'objet de deux réunions de la section québécoise du Comité canadien d'histoire des femmes et des écrits comme ceux de Joan Scott ont laissé leur marque. Joan Scott, Gender and the Politics of History (New York, Cambridge University Press, 1988). 
succédé à «La femme» idéalisée. Quoique soumises aux mêmes structures d'oppression, elles diffèrent cependant selon leur classe sociale, leur origine ethnique, leur orientation sexuelle ou leur appartenance religieuse. Les spécialistes en histoire des femmes, méfiantes envers un universalisme qui les avait si longtemps fondues dans la catégorie «homme», n'ont pas été prises au dépourvu par les défis du postmodernisme et ont très tôt accueilli l'ouverture à la diversité et aux autres groupes sociaux, tout en gardant une distance critique envers certains tenants du poststructuralisme. Ayant pourfendu la domination masculine, les féministes étaient préparées à dénoncer l'ethnocentrisme de leur discipline ou l'essentialisation des peuples autochtones et des communautés culturelles. Alors que l'histoire des femmes profitait des apports des féministes des autres disciplines, elle enrichissait l'histoire dans toutes ses spécialisations.

Le but de cet article n'est pas tant de savoir si l'histoire des femmes a influencé la pratique historienne au Québec que d'identifier l'intégration d'un nouveau sujet et les manifestations de l'approche féministe ${ }^{21}$ dans la recherche et l'enseignement. Une première évidence s'impose: l'inclusion de la variable sexe/genre ${ }^{22}$. Les grandes synthèses, tout comme les monographies, reconnaissent que les données, par exemple sur l'espérance de vie ou sur la participation au marché du travail, sont sexuées. Il est désormais acquis que l'expérience d'un genre ne s'applique pas nécessairement à l'autre: le rapport aux autochtones, les conflits militaires ou la modernisation du Québec n'ont pas été vécus de la même façon par les femmes ou par les hommes.

Dans une deuxième étape, l'histoire des femmes change le paradigme dans lequel s'inscrit et s'écrit l'histoire en suscitant des interrogations inédites sur l'identité et sur les structures d'oppression.

21. Il ne faudrait pas confondre histoire des femmes et histoire féministe. On peut imaginer une histoire des femmes qui ne serait pas féministe comme une histoire du travail qui ne s'inspirerait pas du marxisme, mais il reste que dans l'historiographie québécoise des vingt dernières années, l'histoire des femmes s'est faite dans une perspective féministe.

22. Sexe désigne ici la catégorie biologique et genre la catégorie sociale, ce qui est construit. Depuis quelques années, on assiste au développement de l'histoire des genres, du moins dans la recherche. Cette histoire est, d'une part, plus étroite que l'histoire des femmes, puisqu'elle n'étudie que le construit, et, d'autre part, plus large parce que, logiquement, elle considère les deux genres. Il reste que l'histoire des femmes qui étudie les rapports de pouvoir doit tenir compte du masculin. 
L'histoire des femmes a posé des défis à l'histoire générale en élargissant l'objet historique et en poussant l'histoire à intégrer ces nouveaux objets d'enquête: la socialisation, la reproduction, la sexualité, le travail non rémunéré, pour n'en nommer que quelques-uns. L'histoire $d u$ Québec contemporain de Paul-André Linteau, René Durocher et JeanClaude Robert, dont le premier volume parut en 1979, a montré la voie en donnant une place aux expériences féminines ${ }^{23}$. Aujourd'hui non seulement les auteurs pourraient-ils puiser dans des travaux de recherche plus nombreux, mais les femmes y seraient sans doute présentes différemment. Jusqu'à maintenant, seule Susan MannTrofimenkoff, dans Visions nationales, nous a fourni un exemple de l'intégration des femmes à l'analyse du nationalisme et du cléricalisme québécois ${ }^{24}$.

Pour des raisons particulières au Québec, on devait s'attendre à ce que l'histoire nationale accueille, puis offre une perspective incluant la moitié de la population. En effet, les groupes à statut minoritaire partagent souvent des traits communs; on les trouve ici à l'intersection de l'histoire du Québec et de l'histoire des femmes. La société québécoise est doublement minoritaire en Amérique et au Canada; de leur côté, les femmes, quoique statistiquement majoritaires, possèdent, par leur statut civil et économique, les caractéristiques d'un groupe minoritaire. Tous deux, le Québec et les femmes, font l'objet d'étiquettage, de préjugés, quand ce n'est pas d'exploitation de la part du groupe dominant: le stéréotype de la «priest ridden province» a son équivalent dans celui de «l'instinct maternel féminin». La révision de l'histoire québécoise devait tenir compte de la révision/re-vision, voire de la création de l'histoire des Québécoises.

Malgré son apparition récente sur la scène du monde savant, l'histoire des femmes n'a cessé de se reconceptualiser et de se remettre en question. Les premiers jalons posés relativement à la compréhension d'un problème - soit la contribution des femmes à l'économie familiale, le rôle des religieuses ou l'interconnection des sphères privée et publique - ont vite servi de tremplins pour réviser et raffiner les interprétations antérieures, nécessairement partiales et perfectibles. Il s'ensuivit une ouverture à la révision, gage de souplesse qui constitue un encouragement constant à l'approfondissement des connaissances dans tous les domaines de l'histoire.

23. Paul-André Linteau, René Durocher et Jean-Claude Robert, L'histoire du Québec contemporain (Montréal, Boréal Express, 1979, 1982), 2 vol.

24. Susan Mann-Trofimenkoff, The Dream of Nation: a Social and Intellectual History of Quebec (Toronto, Macmillan, 1982), traduit sous le titre Visions nationales: une histoire $d u$ Québec (Saint-Laurent, Éditions du Trécarré, 1986). 
Le Québec s'est mis à l'étude des groupes sociaux, et depuis une vingtaine d'années, les spécialisations se sont multipliées: histoire ouvrière, histoire de la famille, histoire ethnique, histoire appliquée, histoire des sciences et autres, qu'on retrouve au programme des congrès des sociétés savantes. L'histoire de la famille et l'histoire du travail sont celles qui se sont le plus croisées avec l'histoire des femmes. Longtemps, l'histoire de la famille n'a tenu compte des femmes que pour leur contribution démographique. Plus récemment, on s'est penché sur le rôle des femmes, épouses, mères, veuves ou célibataires, dans le système familial, ainsi que sur les structures légales et les conditions qu'elles imposent aux différents membres de la famille ${ }^{25}$. Le renouvellement des problématiques en histoire de la famille doit beaucoup aux féministes.

L'histoire de la classe ouvrière et l'histoire des femmes, toutes deux tributaires d'une impulsion idéologique - marxiste pour l'une, féministe pour l'autre -, n'ont pu s'ignorer et les contacts ont été féconds. Le travail féminin est indissociable de l'histoire des femmes et de la condition féminine. La place des femmes dans les syndicats, dans les différentes professions, dans la politique de classe ne peut plus être négligée par les spécialistes du travail et de la classe ouvrière. Plus encore, la définition même de travail s'est élargie pour comprendre le travail non rémunéré, le travail domestique, industriel ou ménager, le travail des religieuses, le bénévolat ${ }^{26}$. À des degrés divers, l'histoire des femmes a atteint toutes les autres spécialisations. On est désormais moins naïf en abordant le passé: il est pluriel, il est sexué. Le genre est maintenant une variable inéluctable pour l'appréhender ${ }^{27}$.

À la fine pointe de la recherche, les congrès et les colloques témoignent de l'impact des nouveaux champs de connaissance sur toute la discipline. À la fin des années 1970, on remarque les premières

25. Bettina Bradbury, Working Families: Age, Gender and Daily Survival in Industrialising Montreal (Toronto, McClelland \& Stewart, 1993), traduit par Christiane Teasdale, Familles ouvrières à Montréal: âge, genre et survie quotidienne pendant la phase d'industrialisation (Montréal, Boréal, 1995). France Parent, Entre le juridique et le social: le pouvoir des femmes à Québec au XVII siècle (Québec, GRMF, Université Laval, 1991).

26. Parmi les plus récents ouvrages en histoire des femmes, mentionnons Nadia FahmyEid et al., Femmes, santé et profession. Histoire des diététistes et des physiothérapeutes au Québec et en Ontario, 1930-1980. L'affirmation d'un statut professionnel (Montréal, Fides, 1997). Des sociologues ont étudié, dans une perspective historique, le travail des religieuses. Danielle Juteau et Nicole Laurin, Un métier et une vocation. Le travail des religieuses au Québec de 1901 à 1971 (Montréal, Les Presses de l'Université de Montréal, 1997).

27. Voir, sur ce sujet, l'article de Sylvia Van Kirk, «What Has the Feminist Perspective Done for Canadian History?», dans Ursula Franklin, dir., Knowledge Reconsidered: a Feminist Overview (Ottawa, Canadian Institute for the Advancement of Women, 1984). 
communications en histoire des femmes au congrès de l'IHAF. Si les voies de diffusion parallèles, colloques et revues féministes, restent essentielles à la poursuite des débats, on constate que l'histoire des femmes constitue le thème principal de plusieurs congrès, par exemple sur le travail féminin (RCHTQ), sur l'histoire de Montréal (ACFAS) et, plus récemment, au congrès de l'IHAF en 1996.

Les organismes subventionnaires ont reconnu que la recherche ne peut plus se limiter à une interprétation indifférenciée et, au fond, hégémonique, de la notion d'Homme. Non seulement des projets de grande envergure ayant les femmes pour sujets bénéficient-ils désormais des fonds publics, mais les demandeurs de subventions ne peuvent plus prétendre universaliser à partir du masculin. Si les femmes sont absentes d'un projet, il faut souvent le justifier ${ }^{28}$.

L'enseignement reflète, avec un décalage plus ou moins marqué selon le zèle de l'enseignant, l'évolution de la recherche. Il faut distinguer trois catégories de cours: d'abord les cours en histoire des femmes, généraux ou spécialisés: les femmes de la Nouvelle-France à nos jours ou le travail féminin au $\mathrm{XIX}^{\mathrm{e}}$ siècle, par exemple. Puis les grandes synthèses, telle l'histoire du Québec depuis la Confédération et, enfin, l'histoire spécialisée, comme celle du mouvement ouvrier ou de l'éducation ${ }^{29}$. Le premier groupe est habituellement entre les mains d'historiennes ouvertes à une perspective féministe, mais, dès leur apparition, ces cours en histoire des femmes ont soulevé des interrogations sur la ghettoïsation des femmes et sur le danger de n'être qu'une mode éphémère.

Le confinement de la problématique femmes à certains cours devait-il dispenser les autres collègues d'en parler? L'effet eut été pervers. Tout comme les cours en histoire du mouvement ouvrier ou de l'immigration n'ont pas entraîné l'absence de ces thèmes dans d'autres cours, l'enseignement sur les femmes ne devrait pas résulter en un appauvrissement, bien au contraire. Toutes les matières devraient absorber les nouvelles connaissances en histoire des femmes. Pour saisir les dimensions véritables de la présence de l'histoire des femmes, il faut donc faire état de l'enseignement qui n'a pas le mot «femme» dans le titre. Car si l'on peut compter sur les spécialistes en histoire des femmes pour inclure celles-ci dans le syllabus de leurs autres

28. Il ne s'agit pas ici d'une affirmation gratuite, mais d'une constatation à partir de commentaires d'évaluateurs et d'évaluatrices, ainsi que des membres des jurys, du Conseil de recherches en sciences humaines du Canada et du Fonds pour la formation des chercheurs et l'aide à la recherche (FCAR).

29. On pourrait aussi ajouter l'enseignement multi ou pluridisciplinaire, mais je tiens à me limiter aux cours d'histoire proprement dits. 
cours, ce sont les non-spécialistes qu'il est impérieux de rejoindre. Voilà pourquoi les deux autres groupes, cours de synthèse et cours spécialisés, se montrent plus révélateurs de la prise en considération de l'histoire des femmes.

On ne peut avoir l'indiscrétion d'assister aux cours de ses collègues et les plans de cours ne rendent pas toujours justice à leur contenu. Les grandes synthèses doivent constamment se ressourcer à la lumière des développements empiriques et théoriques des sujets traités. Certains professeurs et professeures de cours généraux se sont méfiés d'une mode temporaire et se sont demandés jusqu'où iraient les additions: une année, leur cours est remanié pour faire place à la classe ouvrière, une autre année pour reconnaître les communautés ethniques, une autre enfin pour admettre les femmes. Et la session n'a toujours au plus que quinze semaines. Pourtant, cette vision à peine caricaturale pose un faux problème. Les femmes ne constituent pas un groupe social «comme les autres»: moitié du genre humain, elles ne peuvent être confinées à une heure de cours sur le suffrage ou sur le travail en temps de guerre. C'est seulement dans l'intégration de la variable femme - pour tenir compte de sa présence ou pour expliquer son absence - que l'on peut constater un véritable impact de la recherche féministe des dernières décennies. Ainsi, l'enseignante ou l'enseignant influencés par l'historiographie féministe n'aurait même pas besoin de singulariser les femmes en leur accordant quelques heures dans la matière au programme, puisqu'elles feraient partie intégrante du passé à l'étude.

Les plus récents plans de cours révèlent en fait que de plus en plus d'historiens et d'historiennes, parfois à la remorque de leurs étudiantes, parfois de leur propre initiative, enrichissent leurs propos des apports de l'histoire des femmes: les travailleurs sont aussi des travailleuses, les immigrants ont deux genres, les guerres impliquent les femmes, elles sont partout et les enseignants qui l'ignorent le font à leurs dépens et aux dépens de la qualité de leur enseignement. Il est toutefois regrettable que persiste encore au sein de trop de syllabus une compartimentation qu'il faut souhaiter n'être que temporaire. On parle beaucoup moins de ghettoïsation aujourd'hui, probablement en raison de la prolifération des cours spécialisés. Si l'immigration ou la religion font partie des cours de synthèse, il y a quand même des cours en histoire de l'immigration ou de la religion. On ne peut non plus parler d'engouement passager pour un champ historique en expansion continue qui affecte tous les domaines d'une discipline. 
Ai-je décrit jusqu'ici comment l'histoire des femmes a influencé la démarche historique au Québec ou, plutôt, comment elle pourrait le faire? Les changements sont-ils illusoires ou les retrouve-t-on bien ancrés dans l'enseignement et concrétisés dans les publications? En 1991, Nadia Fahmy-Eid n'a-t-elle pas déploré «[...] l'insuffisance des échanges et des discussions auxquels les résultats de cette recherche [sur l'histoire des femmes] donnent lieu dans l'ensemble de la communauté historienne - soit en dehors du groupe restreint des historiennes engagées dans ce champ ${ }^{30} »$ ? Sept ans plus tard, le constat doitil demeurer si sévère? Enseignement, recherche, diffusion, reconnaissance institutionnelle marquent à l'évidence des changements tangibles, mais c'est chez les non-spécialistes qu'il faut prendre la mesure des acquis et des déficits à combler.

En général, dans une optique sans doute optimiste, on constate que quelques historiens et historiennes ont pris conscience de la partialité de leur vision et, en dehors de l'histoire des femmes proprement dite, des publications récentes témoignent de cette conscientisation ${ }^{31}$. Si l'intégration est maintenant plus ou moins timidement amorcée, force est de constater que de trop nombreux collègues restent encore réfractaires aux nouvelles problématiques et aux développements des connaissances tant empiriques que théoriques. Il s'agira peut-être toujours autant d'une question de génération que de curiosité ${ }^{32}$.

L'influence de l'histoire féministe sur la redéfinition de l'histoire traditionnelle se retrouve au niveau des interrogations. Les débats sur la reproduction ou sur les stratégies de survie de la classe ouvrière en sont des exemples. L'articulation de la recherche en histoire des femmes aux autres champs de spécialisation en histoire, l'importance de l'interdisciplinarité dans les études féministes et le décloisonnement

30. Nadia Fahmy-Eid, «Histoire, objectivité et scientificité. Jalons pour une reprise du débat épistémologique», loc. cit., 33-34.

31. Il convient de signaler, à titre d'exemple, l'histoire régionale de l'AbitibiTémiscamingue qui tient compte de la contribution et même de l'expérience des femmes. Odette Vincent, dir., Histoire de l'Abitibi-Témiscamingue (Québec, Institut québécois de recherche sur la culture, 1995).

32. Dans son article sur l'histoire québécoise révisionniste des dernières décennies, publié en 1992, l'historien Ronald Rudin a néanmoins construit son argument sur une sélection d'ouvrages qui ignoraient la production en histoire des femmes, comme si les historiennes ne méritaient toujours pas de figurer parmi les «most important members of the generation that emerged on the scene in the 1970s», 35. L'inclusion de ces travaux l'aurait peut-être amené à affiner son analyse. Ronald Rudin, «Revisionism and the Search for a Normal Society: a Critique of Recent Quebec Historical Writing», Canadian Historical Review, 73,1 (mars 1992): 30-61. En mars 1997, un colloque international sur les intellectuels, organisé par le Centre interuniversitaire d'études québécoises de l'Université du Québec à Trois-Rivières, a complètement ignoré la question des femmes intellectuelles. 
des sciences sociales provoquent des interrogations et des comparaisons inédites qui éclairent des aspects jusque-là occultés par l'histoire traditionnelle. Ainsi, loin de marquer un morcellement de la discipline historique pouvant se prêter à une interprétation négative, l'histoire des femmes apporte un gage d'enrichissement et de dynamisme à l'ensemble de la discipline.

Dans le présent contexte économique et devant la vogue de l'idéologie néo-libérale et des contraintes qu'elle impose aux activités intellectuelles, qualifiées de non rentables, il y a lieu de s'inquiéter de l'avenir de l'enseignement dans un champ aussi récent que l'histoire des femmes. Alors qu'on veut sabrer dans les postes et dans les cours «trop» spécialisés, il est crucial que l'histoire de la moitié du genre humain soit intégrée dans les synthèses et les cours plus généraux. Après avoir évité le piège de la ghettoïsation, faut-il maintenant s'inquiéter de la menace d'un éventuel évincement? 